\title{
NUMBER PLATE RECOGNITION USING OCR TECHNIQUE
}

\author{
Er. Kavneet Kaur ${ }^{1}$, Vijay Kumar Banga ${ }^{2}$ \\ ${ }^{1,2}$ E.C.E Department, A.C.E.T, Amritsar, Punjab, India, er.kavneet@gmail.com,vijaykumar.banga@gmail.com
}

\begin{abstract}
Automatic Number Plate Recognition (ANPR) is a special form of Optical Character Recognition (OCR). ANPR is an image processing technology which identifies the vehicle from its number plate automatically by digital pictures. In this paper we have presented an algorithm for vehicle number identification based on Optical Character Recognition (OCR). OCR is used to recognize an optically processed printed character number plate which is based on template matching. This algorithm is tested on different ambient illumination vehicle images. OCR is the last stage in vehicle number plate recognition. In recognition stage the characters on the number plate are converted into texts. The characters are then recognized using the template matching algorithm.
\end{abstract}

Index Terms: Automatic Number Plate Recognition (ANPR), Optical Character Recognition (OCR), Template Matching

\section{INTRODUCTION}

Number plate recognition is a form of automatic vehicle identification. A number plate is the unique identification of vehicle. It is an image processing technology used to identify vehicles by their own number plates. Real time number plate recognition plays an important role in maintaining law enforcement and maintaining traffic rules. It has wide applications areas such as toll plaza, parking area, highly security areas, boarder's areas etc. Number plate recognition is designed to identify the number plate and then recognize the vehicle number plate from a moving vehicle automatically. Automatic number plate recognition has three major parts: vehicle number plate extraction, character segmentation and Optical Character Recognition (OCR). Number plate extraction is that stage where vehicle number plate is detected. The detected number plate is pre-processed to remove the noise and then the result is passed to the segmentation part to segment the individually characters from the extracted number plate. The segmented characters are normalized and passed to an OCR algorithm. At last the optical character information will be converted into encoded text. The characters are recognized using Template matching. The final output must be in the form of string of characters.

\section{RELATED WORK}

Optical Character Recognition (OCR) is widely used technology which converts scanned images of printed text, handwritten text characters into machine encoded text information such as ASCII. It can be recognized printed characters and handwritten characters but the performance is directly dependent from the quality of input documents. The OCR performed offline.
K. K. Kim et. al. [1] is strictly designed for Korean plates. It has designed a system implementing for Support Vector Machines and report impressive average character recognition. M.A. Ko et. al., T. Naito et. al.[2] [3] presents most optical character recognizers introduced in a 2D-plane are able to maintain high success rate only within a limited range of visual angle and shooting distance.

X. Pan et. al. [4] proposed a two stage hybrid recognition system combining statistical and structural recognition method. This work includes Distinguishing similar characters by local structural features and developing a system architecture combining statistical and structural recognition methods. First, the four sub-classifiers independently recognize the character and then recognition results are combined using the Bayes method. Secondly, if the recognized characters belong to the sets of ambiguous characters the structural stage is used for further decision.

Y. Huang et. al. [5] presents to detect the number plate in the acquired image captured from video camera. The gradient operator is used to locate the probable number plate area, the otsu method used to binarize the image and the template matching for recognition. The root-mean-squared-error (RMSE) used for calculating similarity of a prototype and binary image.

Parul Shah et. al. [6] presents a novel algorithm for vehicle chassis number identification based on OCR using artificial neural network. This method gives considerably high value for correct identification rate along with zero wrong identification rate.

Muhammad Tahir Qadri et. al. [7] presents automatic vehicle identification system. The OCR methods in this are sensitive to misalignment and to different sizes. 
S. Hamidreza kasaei et. al. [8] presents a real time and robust method for car number plate detection and recognition. Morphological operator is used to locate the number plate and template matching for character recognition.

Xiaojun Zhai et. al. [9] presented an Artificial Neural Network based OCR algorithm for ANPR applications. The proposed algorithm can meet the real-time requirement of an ANPR system.

\subsection{Number Plate Extraction}

The captured image is in capital RGB format. It is converted into grayscale image and into binary image.

\subsection{Character Segmentation}

The character segmentation part further segments the character individually from the extracted number plate. From input image the first process will be to crop out the number plate characters from starting to the ending point leaving all the extra wide spaces from top to below and from right to left as it is. Characters are equally fit in the plate region. For easy comparison of the input character with the character in the data base the result is normalized into the character set as the size of the images in the database.

\subsection{Optical Character Recognition}

The optical character recognition is a recognition method in which the input is an image and the output is string of character. OCR is a process which separates the different characters from each other taken from an image. Template matching is one of the approaches of OCR. The cropped image is compared with the template data stored in database. OCR automatically identifies and recognizes the characters without any indirect input. The characters on the number plate have uniform fonts then the OCR for number plate recognition is less complex as compared to other methods.

\subsection{Template Matching}

Template matching affects the accuracy of Automatic number plate recognition. The Template matching includes following steps shown in figure 1:

\section{PROPOSED ALGORITHM}

In this paper the processes we have going through are as such. The first step is capturing the image approximately 1 meter from the number plate with camera. The purpose is to get a clear image without distortion. The second step is cropping the number plate from captured image. The cropped image is the input for the character recognition. The third step is character recognition. The OCR technique is used to recognize the character. The flowchart of the presented work is given in figure 2:

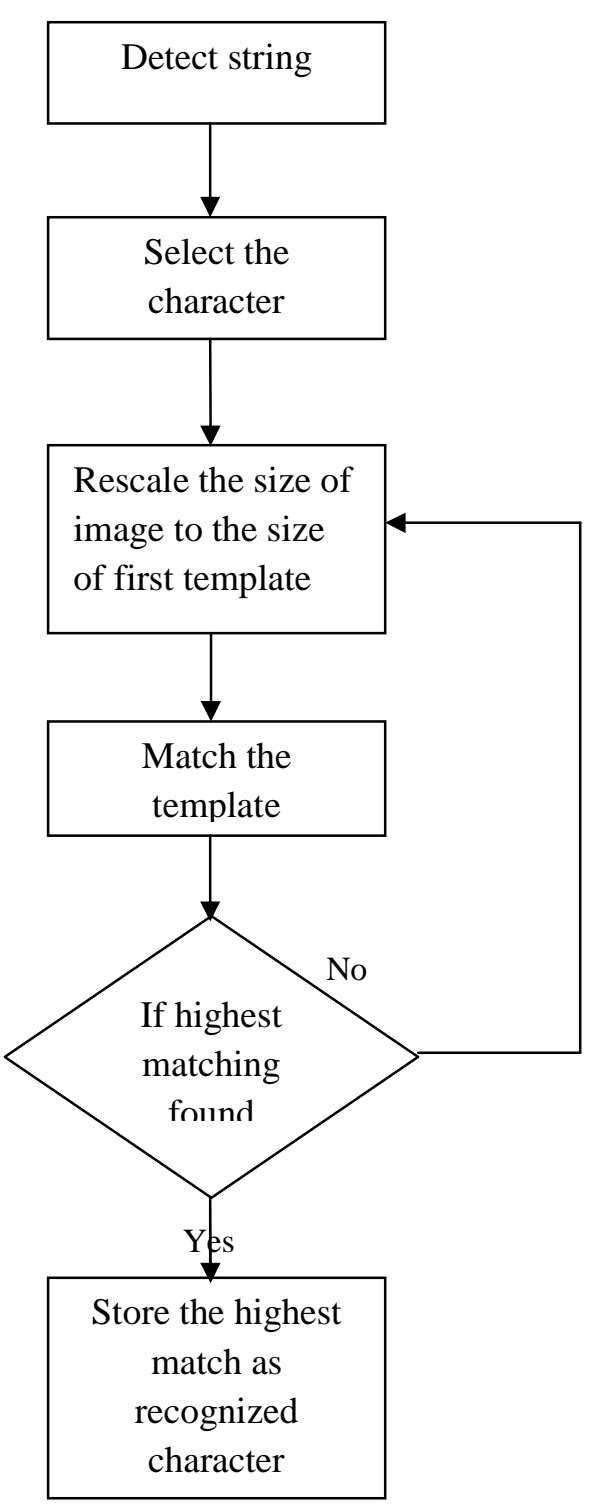

Figure 1: Template matching flow chart

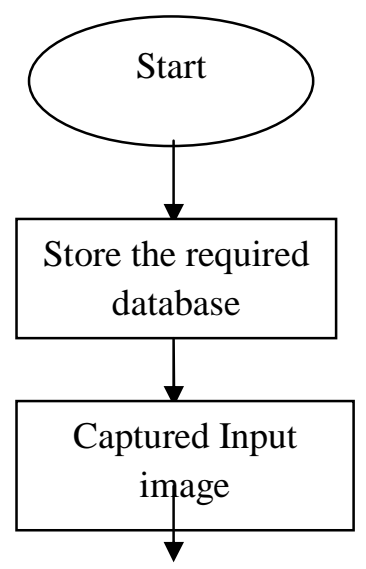




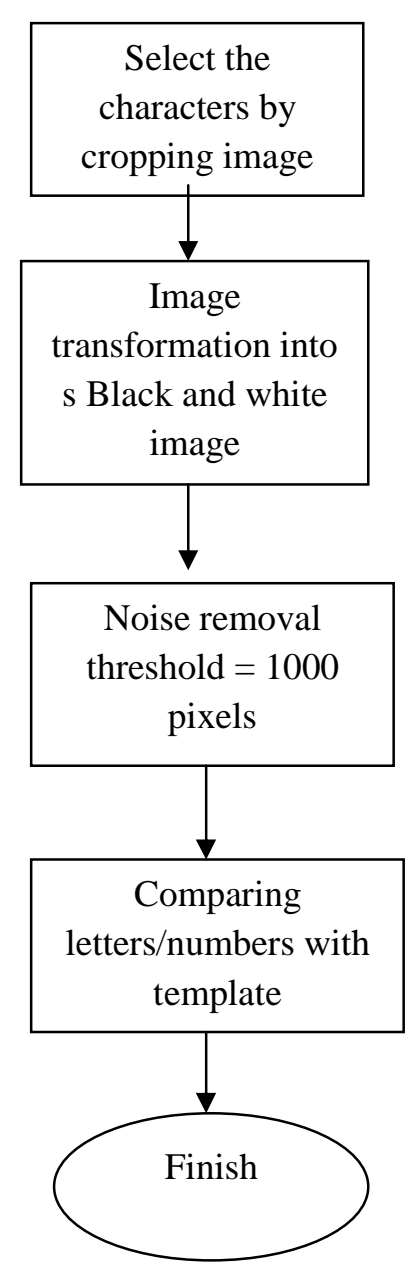

Figure 2: Proposed Algorithm

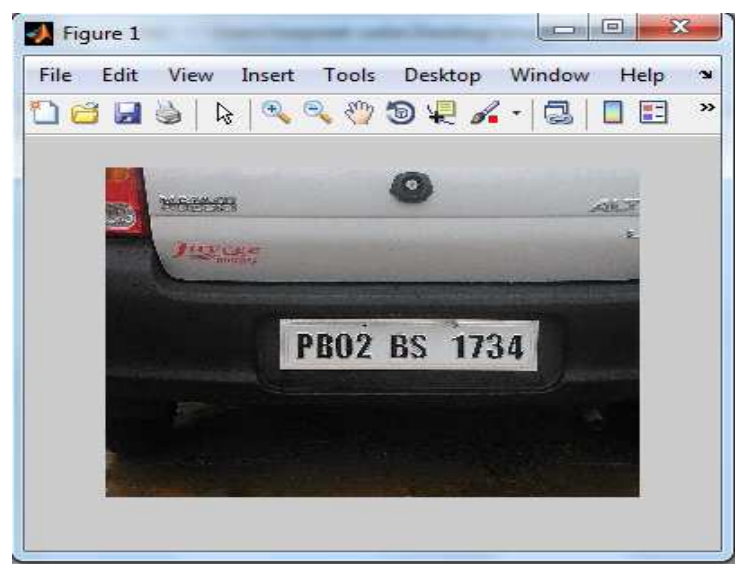

(a).Input Image 1
Figure 1

Fil Ed Vie Ins Ton Desk Winc He $>$
Open File
BD2 BS 1734

(a) Crop Image 1

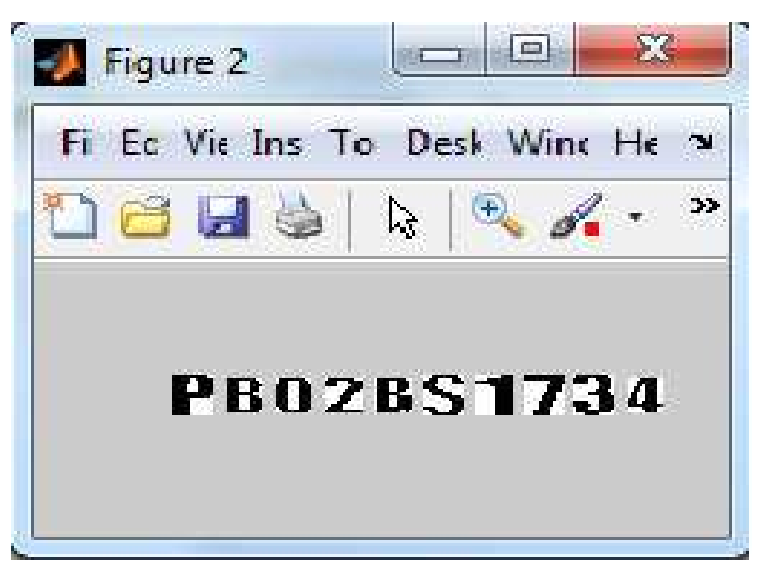

(a) Output Image 1

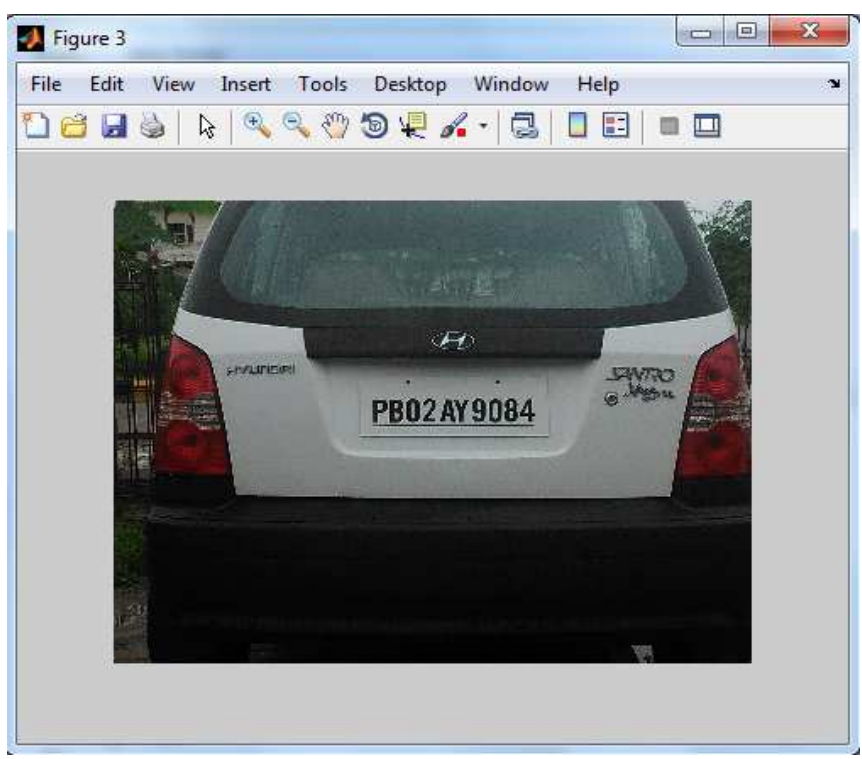

(b) Input Image 2 


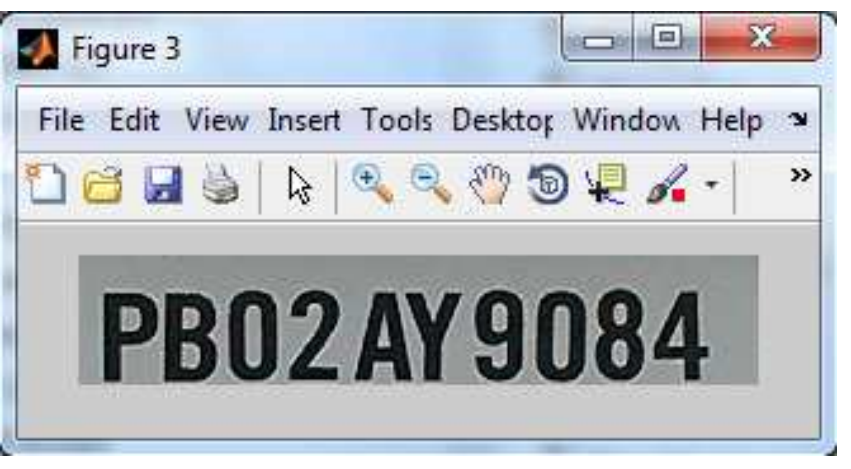

(b) Crop Image 2

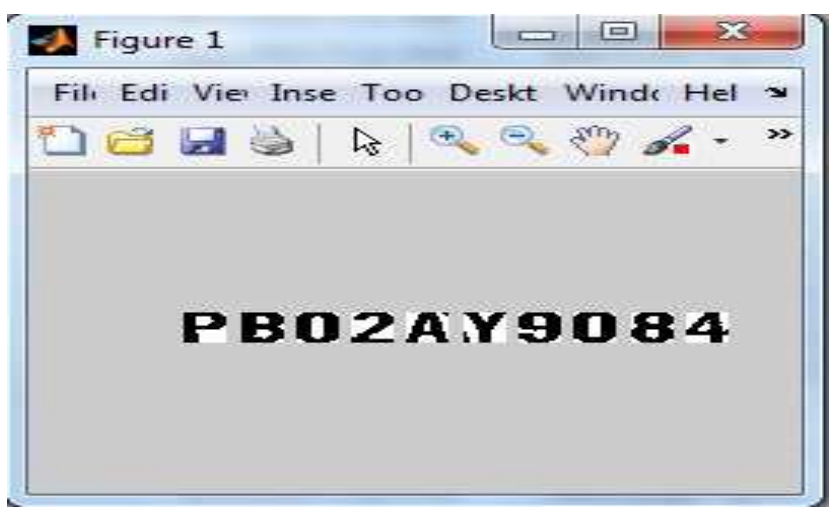

(b) Output Image 2

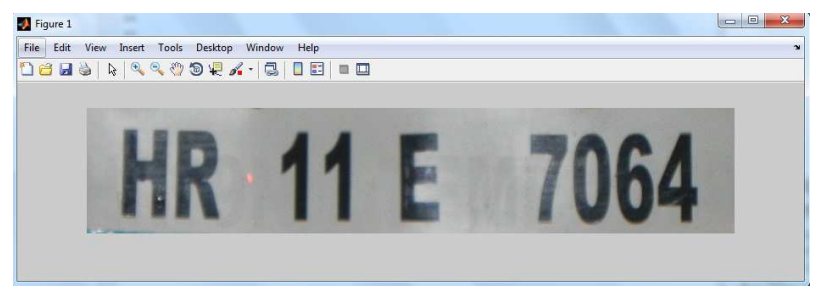

Cropped image

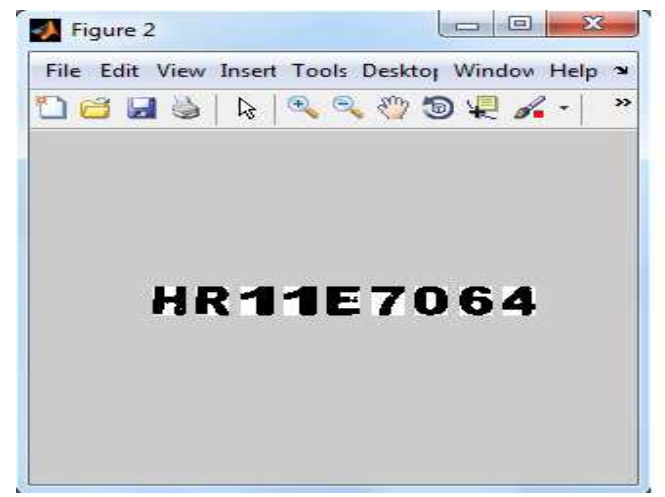

Output Image

\section{CONCLUSION AND FUTURE SCOPE}

In this paper we have checked and evaluated the accuracy of the OCR technique. The Template matching affects the accuracy of number plate recognition. We have found that there are some factors which affect the effectiveness of template matching based on OCR technique i.e. font type, noise in image, tilting etc. In future the work can be done on these factors and efficiency may be increased further for better results.

\section{REFERENCES}

[1] K. K. Kim, K. I. Kim, J. B. Kim and H. J. Kim, “ Learning-based approach for license plate recognition", in Proc. IEEE Signal Process. Soc. Workshop, Neural Network Signal Process. 2000, vol. 2, pp. 614-623.

[2] T. Naito, T. Tsukada, K. Yamada, K. Kozuka, and S. Yamamoto, "Robust License-Plate Recognition Method for Passing Vehicle Under Outside Environment", IEEE Trans. On Vehicular Technology, Vol. 49, No.6, November 2000, pp. 2309-2319.

[3] M.A. Ko and Y.M. Kim, "License Plate Surveillance System Using Weighted Template Matching", in Proc. of the 32nd Applied Imagery Pattern Recognition Workshop, October 2003, pp. 269-274.

[4] X. Pan, X. Ye and S. Zhang, "A hybrid method for robust car plate character recognition", presented at the IEEE International Conference on Systems, Man and Cybernetics, 2004, pp. 4733-4737.

[5] Y. Huang, S. Lai and W. Chuang, "A Template-Based Model for License Plate Recognition", in The 2004 IEEE International Conference on Networking, Sensing \& Control, Taipei,2004,pp. 737-742.

[6] Parul Shah, Sunil Karamchandani, Taskeen Nadkar, Nikita Gulechha, Kaushik Koli, Ketan Lad, "OCR-based ChassisNumber Recognition using Artificial Neural Networks", presented at the IEEE ICVES 2009, pp. 31-34.

[7] Muhammad Tahir Qadri, Muhammad Asif, “ Automatic Number Plate Recognition System for Vehicle Identification using Optical Character Recognition", in IEEE 2009 International Conference on Education Technology and Computer, 2009, pp. 335-338.

[8]S. Hamidreza Kasaei, S. Mohammadreza Kasaei, S. Alireza Kasaei, "New Morphology-Based Method for Robust Iranian Car Plate Detection and Recognition", International Journal of Computer Theory and Engineering, Vol. 2, No. 2 April, 2010 1793-8201. pp. 264-268.

[9] Xiaojun Zhai, Faycal Bensaali and Reza Sotudeh, " OCRBased Neural Network for ANPR", in IEEE 2012. 


\section{BIOGRAPHIES}

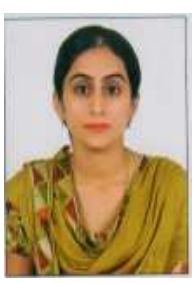

Kavneet Kaur received the B.Tech degree in Electronics and Communication Engineering from PunjabTechnicaUniversity, Jallandhar. Presently, she is working as Lecturer in K.C.E.T, Amritsar as well as pursuing M.Tech in ECE from ACET, Amritsar. Her current research interests include Image Processing.

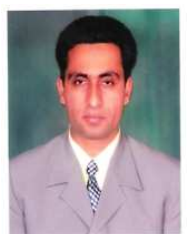

Dr. Vijay Kumar Banga received the B.Tech degree in Electronics and Communication engineering from Punjabi University Patiala, M.Tech degree in Electronics and Communication Engineering from Panjab University handigarh and Phd. From Thapar University Patiala. He is currently working as Principal in Amrisar College of Engineering and Technology, Amritsar. His current research interests include Soft Computing, Image Processing, Autonomous Robots. 